\title{
Abdominal paraaortic paraganglioma: Management of intraoperative hemodynamic emergencies during elective resection procedures (A case presentation)
}

\author{
OVIDIU STIRU $^{1,2}$, ANCA DRAGAN $^{1}$, CRISTINA ADAMACHE $^{1}$, PETRU RAZVAN DRAGULESCU ${ }^{1}$, \\ CARMEN STIRU $^{3}$, ADRIAN TULIN ${ }^{4,5}$, NICOLAE BACALBASA ${ }^{6-8}$, IRINA BALESCU $^{9}$, CAMELIA DIACONU $^{10,11}$, \\ ROXANA CARMEN GEANA ${ }^{1}$, CORNEL SAVU ${ }^{12,13}$ and VLAD ANTON ILIESCU ${ }^{1,2}$
}

${ }^{1}$ Department of Cardiovascular Surgery, 'Prof. Dr. C. C. Iliescu' Institute of Emergency for Cardiovascular Diseases, 022322 Bucharest; ${ }^{2}$ Department of Cardiovascular Surgery, 'Carol Davila' University of Medicine and Pharmacy, 020021 Bucharest; Departments of ${ }^{3}$ Neurology, ${ }^{4}$ General Surgery, 'Prof. Dr. Agripa Ionescu' Clinical Emergency Hospital, 011356 Bucharest; Departments of ${ }^{5}$ Anatomy, ${ }^{6}$ Obstetrics and Gynecology,

'Carol Davila' University of Medicine and Pharmacy, 020021 Bucharest; ${ }^{7}$ Department of Visceral Surgery,

Center of Excellence in Translational Medicine, 'Fundeni' Clinical Institute, 022328 Bucharest;

${ }^{8}$ Department of Obstetrics and Gynecology, 'I. Cantacuzino' Clinical Hospital, 030167 Bucharest;

${ }^{9}$ Department of Surgery, 'Ponderas' Academic Hospital, 021188 Bucharest;

${ }^{10}$ Department of Internal Medicine, 'Carol Davila' University of Medicine and Pharmacy, 020021 Bucharest;

${ }^{11}$ Department of Internal Medicine, Clinical Emergency Hospital of Bucharest, 105402 Bucharest;

${ }^{12}$ Department of Thoracic Surgery, 'Carol Davila' University of Medicine and Pharmacy, 020021 Bucharest;

${ }^{13}$ Department of Thoracic Surgery, 'Marius Nasta' National Institute of Pneumology, 050159 Bucharest, Romania

Received December 24, 2020; Accepted January 26, 2021

DOI: 10.3892/etm.2021.9975

\begin{abstract}
Paragangliomas are extremely rare neuroendocrine tumors. We report a case of a 44-year-old man with hypertension who presented a tumoral mass located retroperitoneally at the aortic bifurcation which included both the common iliac arteries and the posterior left iliac vein, who experienced an unpredictable intraoperative cardiac arrest with electromechanical dissociation at $5 \mathrm{~min}$ after laparotomy. After successful resuscitation and hemodynamic stability, the lesion was fully excised. In the course of tumor manipulation, the patient
\end{abstract}

Correspondence to: Dr Nicolae Bacalbasa, Department of Obstetrics and Gynecology, 'Carol Davila University of Medicine and Pharmacy', 8 Eroilor Sanitari Boulevard, 020021 Bucharest, Romania

E-mail: nicolae_bacalbasa@yahoo.ro

Abbreviations: CRP, C-reactive protein; CK, creatine kinase; CK-MB. creatine kinase myocardial band; CVC, central venous catheter; $\mathrm{CMI}$ cardiac magnetic resonance; ECG, electrocardiogram; LDL, low-density lipoprotein; MAP, mean arterial pressure; TEE, transesophageal echocardiography; TTE, transthoracic echocardiogram; VMA, vanyl mandelic acid

Key words: neuroendocrine tumors, catecholamines, paraganglioma, vanyl mandelic acid, arterial hypertension developed a major hypertensive crisis with peak systolic blood pressure over $280 \mathrm{mmHg}$. Pathologic examination revealed the presence of diffuse proliferation of large and medium-sized mature adipocytes consistent with paraganglioma diagnosis. The patient was discharged at home on the seventh postoperative day. He did not present evidence of recurrence at the one-year follow-up. In conclusion, paragangliomas can require particular management due to their location but also due to their capacity to discharge substances which might induce life-threatening intraoperative complications.

\section{Introduction}

Paragangliomas, like pheochromocytomas, are neuroendocrine tumors originating in the chromaffin cells of the sympathetic system's adrenal medulla or lymph nodes due to their common embryonic origin (1). They can secrete catecholamines, neurotransmitters which are responsible for various symptoms, including diaphoresis, cardiovascular symptoms (hypertension, tachycardia, hypertensive cardiomyopathy), or psychiatric disorders (2). Failure of adequate treatment can lead to acute aortic dissection and cerebral catastrophes $(3,4)$. Genetically, a paraganglioma or a pheochromocytoma may be part of various tumor genetic syndromes associated with other potentially malignant tumors such as multiple endocrine neoplastic syndromes and Von Hippel-Lindau disease (5). In the embryonic period, neuroendocrine ridge cells (the histological origin of pheochromocytoma and paraganglioma) 
migrate from the neural tube to the periaortic region and region of mesenchymal cells that form the fetal cortex of the adrenal gland, forming the medullary adrenal gland (1). Thus, the tumor that develops from the adrenal medulla is called pheochromocytoma, and one that originates in the sympathetic ganglia is called a paraganglioma. Paraganglioma can be located in the cervical, mediastinal, and abdominal sympathetic ganglion chain. It is most often found in patients aged 20-50 years, with a prevalence of 1:10 of the total number of neurotransmitter-secreting neuroendocrine tumors and 1:10 of the total number of malignant paragangliomas with a gender ratio of 1:1 (2). Paragangliomas can be sympathetic, parasympathetic, or dopamine-secreting. Parasympathetic paragangliomas are more common than sympathetic and are most commonly found at the skull base along the cranial nerves IX and X, usually inactive (6). Sympathetic paragangliomas are rare but with a better clinical picture, being secretory, and the rarest form secretes dopamine that occurs due to a deficiency of the enzyme $\beta$-hydroxylase that metabolizes dopamine into norepinephrine (7). This report describes a novel case of a functional paraaortic paraganglioma discovered after laparoscopic cholecystectomy performed due to abdominal pain.

\section{Case presentation}

A 44-year-old male was first examined at the Cardiology Department of a regional hospital. He was admitted after having multiple episodes of hypertension [peak systolic blood pressure (SBP) $>250 \mathrm{mmHg}$ ] immediately after laparoscopic cholecystectomy was performed.

Abdominal computed tomography angiography (CTA) scan revealed a 33x31 mm sized solitary heterogeneous mass at the aortic bifurcation and between the two common iliac arteries (Fig. 1), which was consistent with paraganglioma. The laboratory results showed that plasma metanephrines and normetanephrine were both elevated, respectively, $68.2 \mathrm{pg} / \mathrm{ml}$ (normal local laboratory range $<65 \mathrm{pg} / \mathrm{ml}$ ) and 2,194 pg/ml (normal local laboratory range $<195 \mathrm{pg} / \mathrm{ml}$ ). Serum chromogranin A level was elevated at $189 \mu \mathrm{g} / 1$ (local normal range 27-94 $\mu \mathrm{g} / 1$ ). Simultaneously, urinary vanyl mandelic acid (VMA) was increased at $12 \mathrm{mg} / 24 \mathrm{~h}$ (normal local laboratory range $<8.0 \mathrm{mg} / 24 \mathrm{~h}$ ). The patient was treated with diltiazem $60 \mathrm{mg}$ per day, $5 \mathrm{mg}$ of doxazosin per day, and $25 \mathrm{mg}$ of carvedilol per day; hypertension episodes were managed with a mean arterial pressure (MAP) of $80 \mathrm{mmHg}$. At this point, the patient was referred to our clinic for surgery. Upon admission, the patient was clinically stable with no fever, no peripheral edema, hypertensive with a blood pressure of $145 / 80 \mathrm{mmHg}$ in both upper limbs. The electrocardiogram (ECG) revealed sinus rhythm with a heart rate of 75 beats $/ \mathrm{min}$. The chest $\mathrm{X}$-ray was normal. Laboratory tests revealed a mildly elevated C-reactive protein (CRP) with normal white blood cell count, hypercholesterolemia (222 mg/dl) with low-density lipoprotein (LDL) level of $160 \mathrm{mg} / \mathrm{dl}$, hyperchloremia (109 mmol/l) and creatinine levels of $0.64 \mathrm{mg} / \mathrm{dl}$. Cardiac biomarkers were also mildly elevated with creatine-kinase (CK) of $244 \mathrm{U} / 1$ and creatine kinase myocardial band (CK-MB) of $29 \mathrm{U} / 1$. Transthoracic echocardiography (TTE) performed showed left ventricular hypertrophy with normal ejection fraction. Coronary angiography revealed normal coronary arteries. A

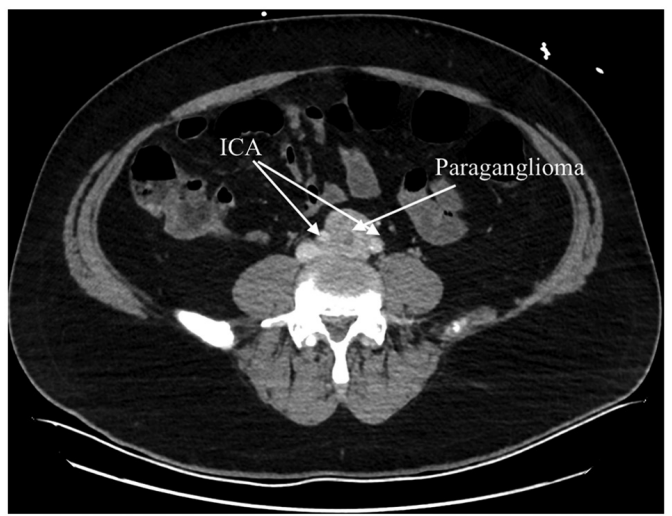

Figure 1. Axial CT scan image showing a round mass, in close contact with the terminal abdominal aorta and both iliac common artery. CT, computed tomography; ICA, iliac common artery.

diagnosis of paraganglioma was confirmed, and surgical treatment was then decided upon.

Routine monitoring included ECG, invasive arterial line (left radial artery), a central venous catheter (CVC) in the left internal jugular vein, dialysis catheter in the right internal jugular vein, 2 peripheral venous catheters $16 \mathrm{G}$, pulse oximetry, temperature, and urinary catheter. An automated external defibrillator and a rapid fluid infuser were available. Rapid-acting cardiovascular drugs were also prepared. Standard preoperative antibiotic prophylaxis was given with cefuroxime.

Extracorporeal membrane oxygenation (ECMO) on standby was prepared. Using the Seldinger technique, both the right common femoral artery and vein were catheterized.

After preoxygenation, general anesthesia was induced with fentanyl $0.5 \mathrm{mg} / \mathrm{kg}$, rocuronium $60 \mathrm{mg}$ and midazolam $5 \mathrm{mg}$ with hemodynamic stability and orotracheal intubation was accomplished. Maintenance of anesthesia was accomplished with fentanyl, rocuronium and sevoflurane. Midline laparotomy incision was performed with an additional dose of fentanyl and rocuronium. Dilatated intestinal loops were observed at gross inspection. To gain access to the retroperitoneal space, the intestines were mobilized (Figs. 2 and 3). Subsequently, the patient experienced severe hypotension and became mildly bradycardic followed rapidly by electromechanical dissociation. Resuscitation protocol was initiated. Chest compressions were started, $2 \mathrm{mg}$ of atropine and $2 \mathrm{mg}$ of titrate intravenous adrenalin were administered with the onset of ventricular fibrillation. A $200 \mathrm{~J}$ electrical shock was used and the patient experienced asystole. Chest compressions were continued and $150 \mathrm{mg}$ of amiodarone, $100 \mathrm{mg}$ of lidocaine $1 \%$ and titrate intravenous adrenalin reaching $20 \mathrm{mg}$ were administered with the reoccurrence of ventricular fibrillation. Another $200 \mathrm{~J}$ shock was used with sinus rhythm establishment at 70 beats/min with systolic blood pressure (SBP) of $120 \mathrm{mmHg}$ with vasopressor support, noradrenaline $50 \mathrm{ng} / \mathrm{kg} / \mathrm{min}$. After $15 \mathrm{~min}$ of cardiac arrest, ST elevation was observed in DII, DIII, aVF and V5-V6 leads. Transesophageal echocardiography (TEE) was performed which showed diffuse hypokinesis of both ventricles. Hemodynamic stability was achieved with the normalization of the ECG and complete remission of hypokinesis $15 \mathrm{~min}$ after the cardiac arrest. 

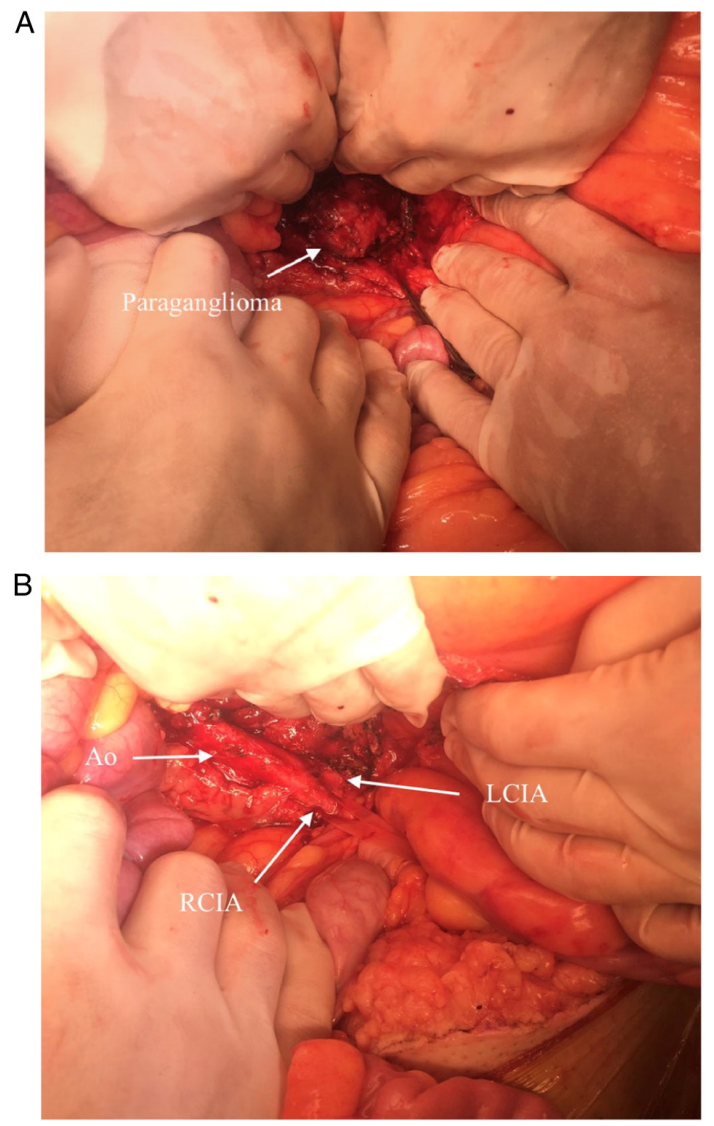

Figure 2. Peroperative view (A) before tumor excision and (B) after tumor excision. Ao, aorta; RCIA, right common iliac artery; LCIA, left common iliac artery.

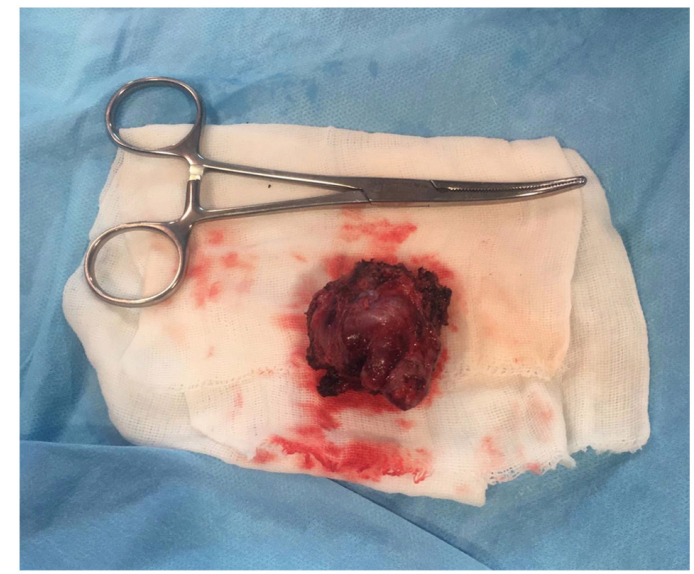

Figure 3. Gross appearance of the retroperitoneal mass.

Considering the hemodynamic stability, there was no need to initiate extracorporeal circulation by ECMO. The serum lactate peaked at $5.4 \mathrm{mmol} / \mathrm{l}$. It was decided by the surgical/anesthesiology/cardiology team to continue the procedure. The retroperitoneal space was opened at the aorta bifurcation. The aorta was cross-clamped at an SBP of $100 \mathrm{mmHg}$. The tumor was identified and the tissue around it was gradually dissected (Fig. 2). During this maneuver, the SBP reached $280 \mathrm{mmHg}$ with 115 beats/min controlled with continuous infusion of urapidil and metoprolol adjusted according to

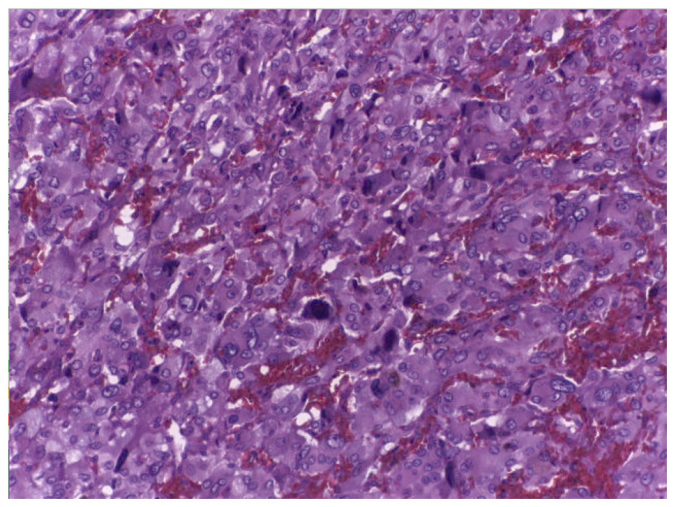

Figure 4. Hematoxylin and eosin stain: Paraganglioma (magnification, x20).

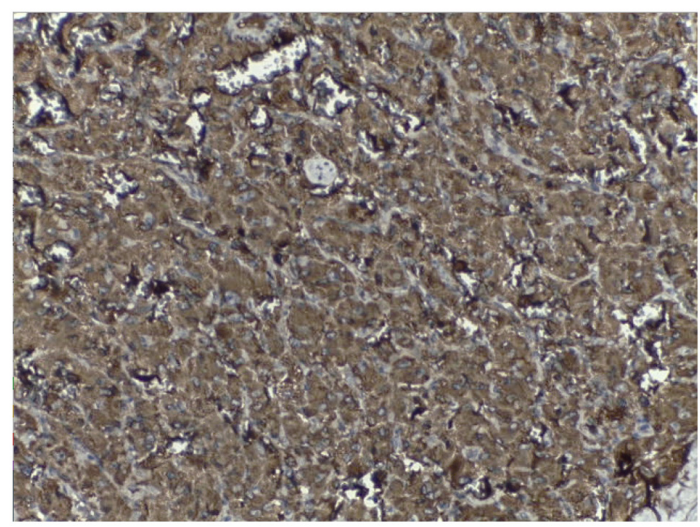

Figure 5. Immunohistochemistry staining showing positivity for chromogranin (magnification, x20).

heart rate reaching an SBP of $140 \mathrm{mmHg}$ and a heart rate of 80 beats $/ \mathrm{min}$, sinus rhythm. The successful removal of the mass was accomplished with no injury to the aorta, iliac arteries or iliac veins (Fig. 3). The clamp was removed and hemodynamic stability was obtained with noradrenaline $100 \mathrm{ng} / \mathrm{kg} / \mathrm{min}$ with a MAP of $70 \mathrm{mmHg}$. The mass was sent for pathology and immunohistochemistry exams. During the procedure, continuous fluid replacement was achieved with 3,500 $\mathrm{ml}$ of Hartmann's solution, $2000 \mathrm{ml}$ of Gelofusine and 5 units of fresh frozen plasma (FFP).

The patient was extubated at $6 \mathrm{~h}$ after admission to the intensive care unit and was weaned off the vasopressor support the next day. No neurologic deficit was observed. Postoperative 24-h urine catecholamine studies and plasma metanephrines and normetanephrine were in the normal range. During the intensive care stay, the patient had a mean blood pressure of $70 \mathrm{mmHg}$ and was transferred to the ward after 2 days of intensive care treatment. Bowel function was recovered on the 4th day. The patient had an uneventful recovery and was discharged on the 7th postoperative day.

The microscopic anatomopathological examination was performed using hematoxylin and eosin staining and showed a highly vascular appearance, with chief cells and sustentacular cells arranged in clusters called a zellballen pattern consistent with a paraganglioma (Fig. 4). Differential diagnosis of paraganglioma from liposarcoma or germ cell tumor was considered. Immunohistochemistry showed strong positivity 
for chromogranin (Fig. 5) and synaptophysin and negativity for vimentin, smooth muscle actin, desmin, pancytokeratin, and S100 which confirmed the diagnosis of paraganglioma.

The patient continued to be followed up; after one year, he did not present with loco-regional recurrence or distant metastasis, and blood pressure was normal without treatment. At six months, 24-h urine catecholamine studies and plasma metanephrines were negative.

\section{Discussion}

Only $15-20 \%$ of chromaffin-cell tumors are paragangliomas (8); $1 \%$ of these tumors are functional and produce catecholamines, the remainder are asymptomatic or cause vague abdominal pain (9). Patients with functional paragangliomas may experience headaches, palpitations, tachycardia, sweating, episodic hypertension, flushing, anxiety, or profuse diaphoresis (6). To the best of our knowledge, this case report is the first to describe the sudden onset of the described symptoms immediately after laparoscopic cholecystectomy was performed. Computed tomography (CT) with contrast provides an initial method for the localization of paragangliomas (sensitivity 88-100\%), and also magnetic resonance imaging (MRI) can be useful when a CT is contraindicated (10). MRI has the highest sensitivity in regards to the detection of extra-adrenal paragangliomas and pheochromocytomas (11). Ten percent of paragangliomas are malignant (12). Approximately 20-42\% of extra-adrenal sympathetic paragangliomas metastasize. The sites of metastasis include lymph nodes, bone, liver, and lung. The survival rate reported at 5-year for metastatic lesions is nearly $36 \%$ (13). Clinical, biochemical, and radiological features, local invasion, and various histopathological features can be suggestive but are inadequate to predict malignancy. In addition, malignancy cannot be reliably diagnosed histologically (14). Surgical excision remains the treatment of choice for resectable paragangliomas (15). Preoperative cardiovascular symptoms must be controlled with alpha and beta-blockers; appropriate patient preparation is crucial to decrease the intraoperative hypertensive spikes and death. Complete surgical excision is the only mode of therapy that has been shown to improve symptoms, and to prolong survival, but, it depends on the tumor's location, and the extent of the involvement of adjacent structures. Therefore, in certain cases complex surgical procedures might be needed; meanwhile improvement in the field of vascular surgery alone or in association with various associated visceral resection might be safely and efficiently performed (15). In our case, involvement was limited to the supra adventitial layer of the aorta and iliac arteries, and we were able to perform a complete resection of the tumor without resection of arteries or veins and without compromising the vascularization of the limbs. Following an R0 resection, patient surveillance requires plasma catecholamines and free metanephrines, urinary levels of catecholamines, VMA, total and fractionated metanephrines. These should be measured every three months during the first year, every six months until the third year, and then annually for up to 10 years (8). CT abdominal scan, abdominal MRI, and scintigraphy performed with 123-I labeled metaiodobenzylguanidine (MIBG) is essential in the assessment surveillance of the patients for metastatic disease, tumor recurrence, or delayed appearance of multiple primary tumors (15). Histopathology examination may reveal chief cells and sustentacular cells arranged in clusters. Chief cells are positive upon immunohistochemistry for synaptophysin, NSE, chromogranin, while sustentacular cells are positive for S-100 protein (16). These findings led to a histological diagnosis of retroperitoneal paraganglioma.

In conclusion, paraaortic paragangliomas pose a therapeutic challenge because of their location, which is in close contact with the aorta, common iliac arteries and veins, and also due to the fact that an $\mathrm{R} 0$ resection must be performed while maintaining intact vascularization of the limbs. Furthermore, management expertise is required when the paraganglioma is a secreting tumor with potentially life-threatening intraoperative complications. Having ECMO protection on standby is advisable in order to deal with unpredictable alterations in blood pressure that can occur during the surgical procedure, and may lead to intraoperative death. In this case, complete surgical resection, normalized plasma catecholamines and urinary levels of catecholamines and VMA, the absence of local and general recurrence one year after the surgical resection are signs that predict a favorable patient outcome.

\section{Acknowledgements}

Not applicable.

\section{Funding}

No funding was received.

\section{Availability of data and materials}

Further information regarding the case presentation is available upon request.

\section{Authors' contributions}

OS, AD, CA and PRD performed the surgical procedure. AD, CA, AT, NB, PRD and CSt reviewed the literature data. AD, $\mathrm{CA}, \mathrm{CD}$ and CSa carried out the preoperative investigation of the patient and intraoperative management. OS, PRD, CS, IB, RCG and NB prepared the draft of the article. VAI was the advisor of the surgical procedures. OS, NB and VAI reviewed the final draft of the manuscript. All authors read and approved the final version of the article.

\section{Ethics approval and consent to participate}

The Ethical Committee of 'Prof. Dr. C. C. Iliescu' Institute of Emergency for Cardiovascular Diseases approved the study.

\section{Patient consent for publication}

Patient consent for publication was obtained and signed by the patient on 16/07/2019.

\section{Competing interests}

There are no competing interests to declare regarding this study. 


\section{References}

1. Lumb R and Schwarz Q: Sympathoadrenal neural crest cells: The known, unknown and forgotten? Dev Growth Differ 57: $146-157,2015$

2. Tabakin AL, Weintraub MA, Radadia KD, Salazar CG, Sadimin E and Singer EA: Metastatic retroperitoneal paraganglioma: Case report and review of the literature. Clin Oncol (Belmont) 4: 1589, 2019.

3. Stiru O, Gorduza EV, Dorobantu FL, Parasca CA, Chioncel O, Bubenek Turconi SI, Filipescu DC and Iliescu VA: Surgical management of type a acute aortic dissection in patients with marfan syndrome: A single center experience. Rev Med Chir Soc Med Nat Iasi 120: 611-618, 2016.

4. Abderrahim SB, Meddeb MA, Marrakchi J, Besbes G, Rammah-Rommani S, Hamdoun M and Khelil MB: Sudden death due to neck paraganglioma: A pediatric case report and review of the literature. Am J Forensic Med Pathol 41: 199-202, 2020.

5. Gaal J, van Nederveen FH, Erlic Z, Korpershoek E, Oldenburg R, Boedeker CC, Kontny U, Neumann HP, Dinjens WN and de Krijger RR: Parasympathetic paragangliomas are part of the Von Hippel-Lindau syndrome. J Clin Endocrinol Metab 94: 4367-4371, 2009.

6. Manger W and Gifford RJ: Pheochromocytoma: A clinical review. In: Hypertension: Pathophysiology, diagnosis, and management. Vol 2. 2nd edition. Laragh J and Brenner B (eds). Raven Press, New York, NY, pp2225-2244, 1995.

7. Feldman JM, Blalock JA, Zern RT, Shelburne JD, Gaede JT, Farrell RE and Wells SA Jr: Deficiency of dopamine-beta-hydroxylase. A new mechanism for normotensive pheochromocytomas. Am J Clin Pathol 72: 175-185, 1979.

8. Samara AA, Diamantis A, Symeonidis D, Anagnostou A, Diamantis AM, Mavrovounis G and Tepetes K: Asymptomatic presacral paraganglioma: Management of an unpredictable intraoperative finding. Surg J (NY) 6: e131-e134, 2020.
9. Mantas D, Kandilis A and Charalampoudis P: Nonfunctioning symptomatic paraganglioma: Is there an optimal follow-up for patients with extra-adrenal benign paragangliomas. J Surg Case Rep 2014: rju092, 2014.

10. Lenders JW, Duh QY, Eisenhofer G, Gimenez-Roqueplo AP, Grebe SK, Murad MH, Naruse M, Pacak K and Young WF Jr: Pheochromocytoma and paraganglioma: An endocrine society clinical practice guideline. J Clin Endocrinol Metab 99: 1915-1942, 2014

11. Archontovasilis F, Markogiannakis H, Dikoglou C, Drimousis P, Toutouzas KG, Theodorou D and Katsaragakis S: Paraganglioma of the greater omentum: Case report and review of the literature. World J Surg Oncol 5: 87, 2007.

12. Plouin PF, Amar L, Dekkers OM, Fassnacht M, GimenezRoqueplo AP, Lenders JW, Lussey-Lepoutre C and Steichen O: European society of endocrinology clinical practice guideline for long-term follow-up of patients operated on for a phaeochromocytoma or a paraganglioma. Eur J Endocrinol 174: G1-G10, 2016.

13. Sclafani LM, Woodruff JM and Brennan MF: Extraadrenal retroperitoneal paragangliomas: Natural history and response to treatment. Surgery 108: 1124-1129, 1990.

14. Mannina EM, Xiong Z, Self R and Kandil E: Resection of a catecholamine-elaborating retroperitoneal paraganglioma invading the inferior vena cava. Case Rep Surg 2014: 837054, 2014.

15. Disick GI and Palese MA: Extra-adrenal pheochromocytoma: Diagnosis and management. Curr Urol Rep 8: 83-88, 2007.

16. Lack EE, Cubilla AL, Woodruff JM and Lieberman PH: Extra-adrenal paragangliomas of the retroperitoneum: A clinicopathologic study of 12 tumors. Am J Surg Pathol 4: 109-120, 1980. 